\title{
New Looks at Capillary Zone \\ Electrophoresis (CZE) and Micellar \\ Electrokinetic Capillary Chromatography (MECC) and Optimization of MECC
}

\author{
Kiumars Ghowsi and Hosein Ghowsi \\ Additional information is available at the end of the chapter
}

http://dx.doi.org/10.5772/45760

\section{Introduction}

The origin of theoretical plates for electrophoresis in general sense has been obtained from the work of Giddings ${ }^{1}$. Giddings has derived the following equation for the number of theoretical plates.

$$
N=\frac{-\Delta \mu^{e x t}}{2 \Theta R T}
$$

where coefficient $\Theta=$ unity when molecular diffusion acts alone and exceed unity when other process contributes. $\mathrm{R}=$ gas constant and $\mathrm{T}=$ temperature and $\mu^{\text {ext }}=$ chemical potential conventionally substution of $\Delta \mu^{e x t}=Z F V$ into eqn.(1) gives for the number of theoretical plates.

$$
N=\frac{Z F V}{2 R T}
$$

For an ideal process, in which $\Theta=1$ and $\mathrm{T}=298 \mathrm{~K}$, this equation reduces

$$
\mathrm{N}=20 \mathrm{ZV}
$$

where we have used the Faraday constant $F=96500$ coulombs/mol. This voltage drops $V$ in the range of $100-10000 \mathrm{v}$ with charge number $\mathrm{Z}=1$ capable of yielding 2000-200000 theoretical plates, a range comparable to that found in chromatographic system. Jorgenson and Lukacs², also in their land mark paper provided a theory for capillary zone 
electrophoresis (CZE) in which they proposed two fundamental equations for resolution and migration time. Resolution and number of theoretical plates are the focus of this work. This is how number of theoretical plates are derived for capillary zone electrophoresis and used in resolution equation by Jorgenson and Lukacs ${ }^{2}$ by starting with Gidding number of theoretical plates.

$$
N=\frac{Z F V}{2 R T}
$$

$\mathrm{V}=$ potential across capillary is substituted by EX where E=electric field and $X=$ length of the capillary. $Z=$ charge number for ion is assumed to be 1 , hence

$$
\begin{gathered}
N=\frac{F F X}{2 R T} \\
X=t\left[v_{e o}+\left(v_{e p}\right)_{A B}\right]
\end{gathered}
$$

Eqn.6 where $\left(v_{e p)_{A B}}=\frac{1}{2}\left[\left(v_{e p}\right)_{A}+\left(v_{e p}\right)_{B}\right]\right.$ is substituted by $\mathrm{X}$ in eq.(5). $\mathrm{t}$ is the time it takes the analyte travels across the capillary and $v_{e o}$ is the electroosmotic velocity $\left(v_{e p}\right)_{A}$ and $\left(v_{e p}\right)_{B}$ are the electrophoretic velocity of two analytes A and B with close electrophoretic velocities. By substitution of equation(6) into equation (5) one obtains

$$
N=\left[\left(\frac{E}{2 R T}\right) E t\left[v_{e o}+\left(v_{e p}\right)_{A B}\right]\right]
$$

There is mistake occurs in Jorgenson and Lukacs work ${ }^{2}, \mathrm{t}$ in eqn. (7) is replaced by eqn.(8), this is the case when electroomosis is absent.

$$
t=\frac{X}{v_{e p}}
$$

By making this mistake substitution eqn (8), what has been obtained by Jorgenson and Lukacs for $\mathrm{N}$, eqn.(2) is

$$
N=\frac{F}{2 R T} E\left[\left(\frac{X}{v_{e p}}\right)\left[\left(v_{e o}\right)+\left(v_{e p}\right)_{A B}\right]\right]
$$

$\mathrm{E}, \mathrm{X}$, is the $\mathrm{V}$, voltage across the capillary and $v_{e p}$ the electrophoretic velocity of the analyte and $v_{e o}$ the electroosmotic flow in the capillary are substituted in eqn.(9) by $\mu_{e p} E$ and $\mu_{e o} E$ where $\mu_{e p}$ and $\mu_{e o}$ are the electrophoretic and electroosmotic mobilities and $\mathrm{E}$ is the electric field. By this substitution the following eqn.(10) is obtained for $\mathrm{N}$ 


$$
N=\left(\frac{F V}{2 R T}\right)\left[\frac{\mu_{e o}+\left(\mu_{e p}\right)_{A B}}{\left(\mu_{e p}\right)_{A B}}\right]
$$

By using Einstain relation eqns.(11) and (12) we substitute for $\mu_{e p}$ from eqn.(12)

$$
\begin{aligned}
& \frac{D}{\mu_{e p}}=\frac{R T}{F} \\
& \mu_{e p}=\frac{F D}{R T}
\end{aligned}
$$

Where $\mathrm{D}$ is the Diffusion constant of the analyte by this substitution and simplification eqn.13 is obtained

$$
N=\frac{V}{2 D}\left[\mu_{e o}+\left(\mu_{e p}\right)_{A B}\right]
$$

This is the equation for the number of theoretical plates the Jorgenson and Lukacs use to obtain the resolution equation. $\mathrm{N}$ is driven by mistake ${ }^{2}$. The resolution equation is given by them

$$
R_{s}=\frac{\left(\mu_{e p}\right)_{A}-\left(\mu_{e p}\right)_{B}}{\mu_{e o}+\left(\mu_{e p}\right)_{A B}} \cdot \frac{\sqrt{N}}{4}
$$

substituting eqn.(13) for $\mathrm{N}$ into eqn.(14), the resolution can be expressed as

$$
R_{s}=\frac{\left(\left(\mu_{e p}\right)_{A}-\left(\mu_{e p}\right)_{B}\right)}{\left(\mu_{e o}+\left(\mu_{e p}\right)_{A B}\right)^{1 / 2}} \cdot \frac{\sqrt{V}}{4 \sqrt{2 D}}
$$

The above eqn.(15) is the same equation that was obtained by Jorgenson and Lukacs².

This equation is incorrect. In the present work equation for resolution which is cosidered correct equation is proposed.

\section{Theory}

According to Gidding's theory ${ }^{1}$ the evaluation of the ultimate capabilities of zone electrophoresis is possible. To calculate the number of theoretical plates and separable zone achieveable in ideal zone electrophoresis, the electrostatic force exerted on a mole of charged particles on electric field of strength $\mathrm{E}$ is

$$
\text { Force }=\mathrm{ZFE}=\mathrm{f}
$$

where $\mathrm{z}=$ net charge of a single particle in proton units and $\mathrm{F}=$ Faraday constant the negative chemical potential drop across the separation path. 


$$
-\Delta \mu^{e x t}=f X^{\prime}
$$

In which $X^{\prime}$ is the distance where $\mathrm{f}$, force applied in capillary eletrophoresis. For conventional mode of capillary zone electrophoresis, electroosmotic and electrophoretic velocities are in opposite direction. This conventional mode is similar to tread mill and electric stairs where the moveing object has two movements one walking and the other one movement of the stairs.

There $X^{\prime}$ in eqn. (17) is not the length of capillary, it is effective distance where the electric force which is applied is greater than the length of capillary is called

$$
-\Delta \mu^{e x t}=f X^{\prime}
$$

Then by substituting eqn.(18) into eqn.(1), the eqn.(19) for the number of theoretical plates results.

$$
N=\frac{f X^{\prime}}{2 R T}
$$

$X^{\prime}=$ effective length which looks like a treadmill, the solute is like a person which can run for miles on the mill but actually he has stayed stationary, $\mathrm{f}=\mathrm{FE}$ force is Faraday constant times electric field. By substituting force in eqn. (19) one gets

$$
N=\frac{F}{2 R T} E X^{\prime}
$$

By definition, the effective distance the analyte travels under the force of electric field, $X^{\prime}$ divided by the relation times, $\mathbf{t}$, is equivalent to electrophoretic $\left(v_{e p}\right)_{A B}$ velocity, $\left(v_{e p}\right)_{A B}$, as the following

$$
\left(v_{e p}\right)_{A B}=\frac{X^{\prime}}{t}
$$

Where $\left(v_{e p)_{A B}}=\frac{1}{2}\left[\left(v_{e p}\right)_{A}+\left(v_{e p}\right)_{B}\right]\right.$

By the help of eqn.(21) $X^{\prime}$ can be substituted into eqn.(20). Now eqn.(22) can be rewritten to include electrophoretic velocity.

$$
N=\left(\frac{F}{2 R T}\right)\left(v_{e p}\right)_{A B} E t
$$

Replacing the electrophoretic velocity variable with the product of electrophoretic mobility and electric field $v_{e p}=\mu_{e p} E$ yields the following expression:

$$
N=\left[\left(\frac{F}{2 R T}\right)\left[\left(\mu_{e p}\right)_{A B} E^{2} t\right]\right.
$$


In order to observe the dependence, efficiency has on capillary length and electroosmosis, a substitution for the time variable in eqn.(21) is made. Net displaccment of the analyte or capillary length, $\mathrm{X}$, is related to the retention time, $\mathrm{t}$ as shown

$$
\left.N=t\left[v_{e o}\right)+\left(v_{e p}\right)_{A B}\right]
$$

Rearragement of eqn.(24) given eqn.(25)

$$
t=\frac{X}{v_{e o}+\left(v_{e p}\right)_{A B}}
$$

substituting eqn.(25) into eqn.(20) yields the expression,

$$
N=\frac{F}{2 R T}\left[\frac{\left(v_{e p}\right)_{A B}}{v_{e o}+\left(v_{e p}\right)_{A B}}\right] X E
$$

By making additional substitution for electrophoretic and electroosmotic velocity produced, $v_{A B}=\left(\mu_{e p}\right)_{A B} E$ and $v_{e o}=\mu_{e o} E$, a final equation for efficiency is

$$
N=\left(\frac{F}{2 R T}\right)\left(\frac{\left(\mu_{e p}\right)_{A B}}{\mu_{e o}+\left(\mu_{e p}\right)_{A B}}\right) X E
$$

plate height is the ratio of effective length $\mathrm{X}^{\prime}$ to efficiency $\mathrm{N}$ is

$$
H=\frac{X^{\prime}}{N}
$$

Substituting equation into eqn.(28) yields an expression for plate height.

$$
H=\frac{2 R T}{F E}
$$

This interesting result shows that the theoretical plate height is independent of electroosmotic flow when it is based on the effective distance the analyte travels rather than the capillary length. Instead, plate height has a simple inverse relation with the electric field strength.

Fig .1 Shows the invers relation between theoretical plate height and electric field strength. Electric field strength is the voltage across two ends of capillary divided by the length of capillary.

Equation for resolution: Based width resolution is the quantitative measure of ability to separate two analytes. For two adjacent peaks with similar elution times, peak showed be nearly identical:

$$
W_{A} \approx W_{B} \approx W_{A B}
$$




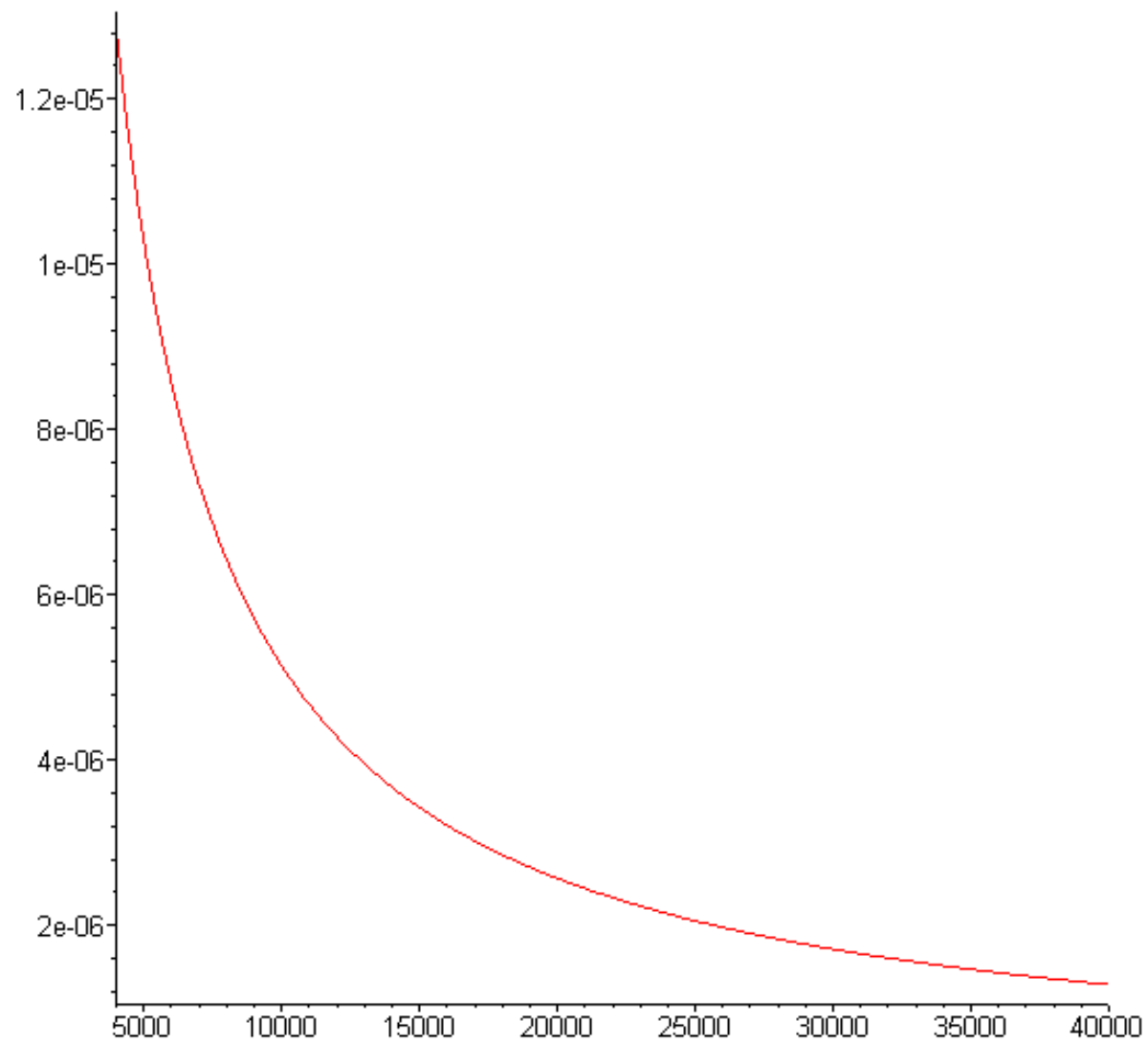

Figure 1. Theoretical plate height versus electric field for CZE.

where $W_{A B}=\frac{1}{2}\left(W_{A}+W_{B}\right)$.

Assuming eqn.(30) is true, resolution for species A and B expressed in terms of their retention times and the peak base width for either species ${ }^{2}$.

$$
R_{S}=\frac{\left(t_{R}\right)_{B}-\left(t_{R}\right)_{A}}{W_{A B}}
$$

The conventional expression for separation can be written with parameters related to either species $\mathrm{A}$ or $\mathrm{B}$, shown here using the retention time and peak base width for species $\mathrm{B}^{2}$.

$$
N=16\left[\frac{\left(t_{R}\right)_{B}}{W_{A B}}\right]^{2}
$$

By combining eqns. (31) and (32) an equation for chromatography is produced that expresses resolution in terms of efficiency and retention time ${ }^{4}$. 


$$
R_{s}=\frac{\sqrt{N}}{4}\left[\frac{\left(t_{R}\right)_{B}-\left(t_{R}\right)_{A}}{\left(t_{R}\right)_{A B}}\right]
$$

The resolution time variable $t_{R}$ and the efficiency $\mathrm{N}$ are eliminated by inserting eqns.(25) and (26) into eqn.(33) for the analyte B:

$$
R=\left[\left(\frac{F}{32 R T}\right)\left(\frac{\left(v_{e p}\right)_{A B}}{v_{e o}+\left(v_{e p}\right)_{A B}}\right) X E\right]^{1 / 2}\left[\frac{\left(v_{e p}\right)_{A}-\left(v_{e p}\right)_{B}}{v_{e o}+\left(v_{e p}\right)_{A B}}\right]
$$

when electrophoretic velocity, $v_{e p}$, is replaced with $\mu_{e p} E$ and applied patential, $\mathrm{V}$ is substituted for XE one obtains the following equation for the resolution:

$$
R_{s}=\left[\frac{F V\left(\mu_{e p}\right)_{A B}}{32 R T}\right]^{1 / 2}\left[\frac{\left(\mu_{e p}\right)_{A}-\left(\mu_{e p}\right)_{B}}{\left(\mu_{e o}+\left(\mu_{e p}\right)_{A B}\right)^{3 / 2}}\right]
$$

In order to make a comparison between new resolution eqn.(35) obtained in present work and Jorgenson Lukacs equation for resolution eqn.(15) could be transformed to the following equation by using Einstein relation relating diffustion constant to mobility $\frac{D}{\mu}=\frac{R T}{F}$ and

$$
R_{s}=\left[\frac{F V}{32 R T\left(\mu_{e p}\right)_{A B}}\right]^{1 / 2}\left[\frac{\left(\mu_{e p}\right)_{A}-\left(\mu_{e p}\right)_{B}}{\left(\mu_{e o}+\left(\mu_{e p}\right)_{A B}\right)^{1 / 2}}\right]
$$

Jorgenson and Lukacs equation (eqn.36) and new derived Ghowsi equation(eqn.38) for resolution are given as

$$
R_{s}=\left[\frac{F V}{32 R T\left(\mu_{e p}\right)_{A B}}\right]^{1 / 2}\left[\frac{\left(\mu_{e p}\right)_{A}-\left(\mu_{e p}\right)_{B}}{\left(\mu_{e o}+\left(\mu_{e p}\right)_{A B}\right)^{3 / 2}}\right]
$$

This interesting observation that for the absence of electrosmotic flow in Jorgenson and Lukacs equation the resolution eqn.36 is converted to

$$
R_{s}=\left[\frac{F V}{32 R T}\right]^{1 / 2}\left[\frac{\left(\mu_{e p}\right)_{A}-\left(\mu_{e p}\right)_{B}}{\left(\mu_{e p}\right)}\right]
$$

The other resolution equation obtained in present work Ghowsi's eqn.(35) with presence of electroosmotic flow $R_{S}$ is converted to

$$
R_{s}=\left[\frac{F V}{32 R T}\right]^{1 / 2}\left[\frac{\left(\mu_{e p}\right)_{A}-\left(\mu_{e p}\right)_{B}}{\left(\mu_{e p}\right)_{A B}}\right]
$$

It is interesting that only for this case when electroosmosis is absent the resolution equation of Jorgenson and Lukacs eqn.(15) with the help of Einstein relation is equal to Ghowsi's derived equation eqn.(38) at present for capillary zone electrophoresis. 


\section{Micellar electrokinetic capillary chromatography (MECC)}

By converting figure of Merits in MECC to electrochemical parameters ${ }^{5}$ and pursuing similar procedure we applied to capillary electrophoresis and using effective length ${ }^{3}$ solute travels rather than length of capillary and then converting the resolution equation in terms of chromatography parameters new equation for resolution could be found which is published in another paper?

\section{Optimization of micellar electrokinetic capillary chromatography (MECC) as a nano separation technique using three dimensional and two dimensional plottings of characteristic equations}

Feyman with the lecture of plenty of room at the bottom at an American Physical Society at Caltech on December 29, 1959 considered the possibility of direct manipulation of individual atoms as a more powerful forms of synthetic chemistry than those used at the time. In conventional chromatography there are two phases involved one is the stationary phase and one is the mobile phase ${ }^{6}$. Terabe et al proposed Micellar Electrokinetic Capillary Chromatography ${ }^{8}$, MECC, which has the smallest pseudo stationary phase within nano range called micelle.

The very high strength of separation comes from these nano sized materials. That is why we call this technique MECC.

\section{Nano separation technique}

There are several work which have been done to final the optimum conditions of this Nano Separation Technique ${ }^{5,8-10}$.

In all optimization characteristic equation is the focus.

What is the characteristic equation?

In column chromatography the resolution equation is given as ${ }^{6}$

$$
R_{s}=\frac{N^{1 / 2}}{4} \cdot \frac{\alpha-1}{\alpha} \cdot \frac{k^{\prime}}{1+k^{\prime}}
$$

Where $\mathrm{k}^{\prime}$ is the capacity factor, $\alpha$ is the selectivity and $\mathrm{N}$ is the number of theoretical plates. Terabe $^{8}$ et al proposal MECC for the first time and they proposed the resolution equation for MECC:

$$
R_{s}=\frac{N^{1 / 2}}{4} \cdot \frac{\alpha-1}{\alpha} \cdot \frac{k^{\prime}}{1+k^{\prime}} \cdot \frac{1-t_{o} / t_{m c}}{1+t_{o} / t_{m c}}
$$

Where $\mathrm{N}, \alpha$ and $\mathrm{k}^{\prime}$ were already defined, a new term is appearing in equation (41) for resolution is $\frac{1-t_{o} / t_{m c}}{1+t_{o} / t_{m c}}$. In this term $\mathrm{k}^{\prime}$ is the capacity factor and $t_{o}$ and $t_{m c}$ are retention 
times of the aqueous and micellar phases respectively. The characteristic equation for MECC to optimize is from equation(41):

$$
f\left(k^{\prime}, t_{o} / t_{m c}\right)=\frac{k^{\prime}}{1+k^{\prime}} \cdot \frac{1-t_{o} / t_{m c}}{1+\left(t_{o} / t_{m c}\right) k^{\prime}}
$$

This characteristic equation has two variables $\mathrm{k}^{\prime}$ and the ratio $t_{o} / t_{m c}$. In a recent work a new model for the MECC using a model based on effective length solute migrated as a similar to tread mill case were proposed ${ }^{7}$. Based an this model

$$
R_{s}=\frac{1}{4}\left[\left(D_{e p}\right)_{B} R T / 4\right]^{1 / 2}\left(N_{\text {pseudo }}^{1 / 2}\right)\left[\frac{\alpha-1}{\alpha}\right]\left[\frac{k^{\prime}}{k^{\prime}+1}\right]^{3 / 2} \cdot \frac{1-\left(t_{o} / t_{m c}\right)}{1+\left(t_{o} / t_{m c}\right) k^{\prime}}
$$

Variables of this equations were defined in the previous work ${ }^{7}$. The characteristic equation of $R_{s}$ for equation (43) is the last two terms.

$$
f\left(k^{\prime}, t_{o} / t_{m c}\right)=\left(\frac{k^{\prime}}{1+k^{\prime}}\right)^{3 / 2} \cdot\left(\frac{1-t_{o} / t_{m c}}{1+\left(t_{o} / t_{m c}\right) k^{\prime}}\right)
$$

In a comparison between the characteristic equation (42) obtained by Terabe ${ }^{8}$ and the characteristic equation obtained based on the new model $^{7}$,equation(44), the difference is the power of $\left(\frac{k^{\prime}}{k^{\prime}+1}\right)$ term where in equation (42) the power of this term is 1 and in equation (44) the power of this term is 3/2. The rest of these two equations (42) and (44) are the same. There are two other characteristic equations need to be obtained. For the first time J. P. Foley obtained an equation for a compromise between resolution and the migration time. He introduced $R_{s} / t_{R} \cdot t_{R}$ is given in Terabe's work ${ }^{8}$ as

$$
t_{R}=\left[\frac{1+k^{\prime}}{1+\left(t_{o} / t_{m c}\right) k^{\prime}}\right] t_{o}
$$

Two characteristic equations for $R_{s} / t_{R}$ are obtained one is for Terabe $R_{s}$ equation (41) and the other one is for $R_{s}$ in equation (43). The two characteristic equations for $R_{s} / t_{R}$ are given as below correspondingly.

$$
\begin{gathered}
f\left(k^{\prime}, t_{o} / t_{m c}\right)=\frac{k^{\prime}}{\left(1+k^{\prime}\right)^{2}} \cdot\left(1-\frac{t_{o}}{t_{m c}}\right) \\
f\left(k^{\prime}, t_{o} / t_{m c}\right)=\frac{k^{\prime}}{\left(1+k^{\prime}\right)^{5 / 2}} \cdot\left(1-\frac{t_{o}}{t_{m c}}\right)
\end{gathered}
$$

\section{Two dimensional and tree dimensional plots of characteristic equations}

In present work by the help of modern technology of computer and three dimensional software of Dplot direct access to the plot of characteristic equations (42) and (44) are possible. 
Figure 2 shows the three dimensional plot of characteristic equation (42) obtained by Terabe 8 et al.

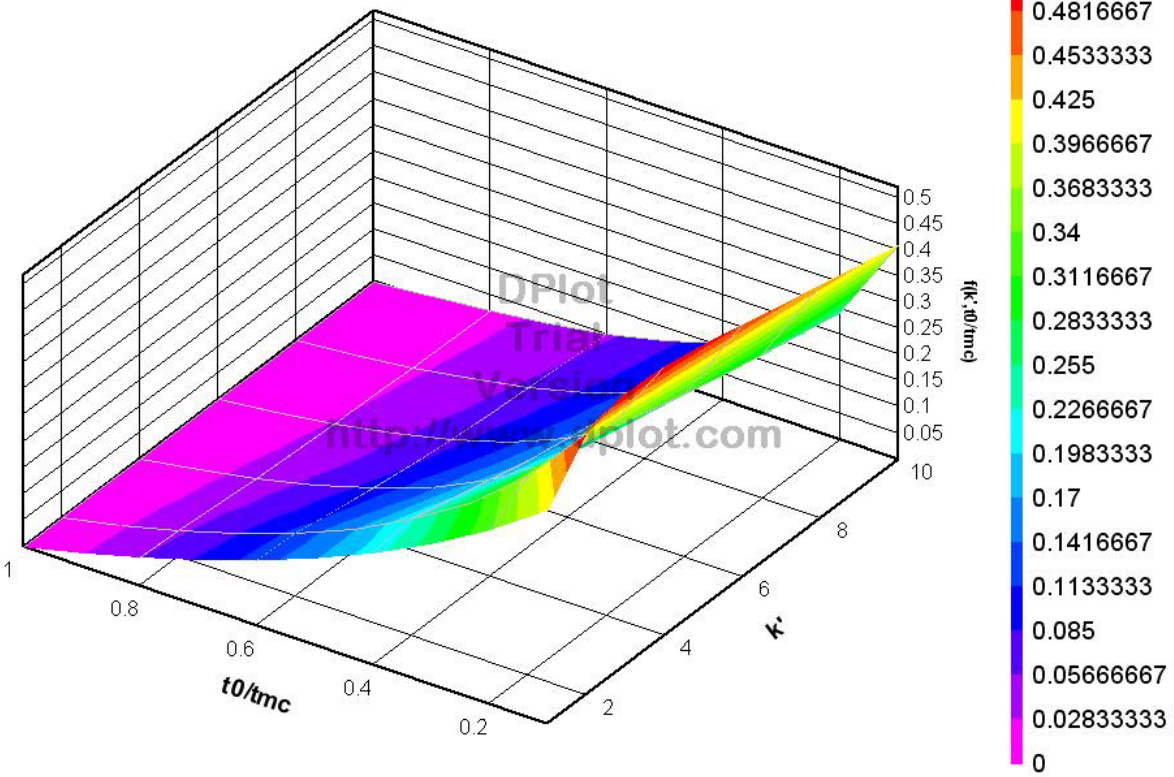

Figure 2. Three dimensional plot of Terabe's characteristic equation for resolution of MECC.

In this work $f\left(k^{\prime}, t_{o} / t_{m c}\right)$ is a function of two independent variables $\mathrm{k}^{\prime}$ and $t_{o} / t_{m c} \cdot \mathrm{k}^{\prime}$ is changing between 1 to 10 and $t_{o} / t_{m c}$ changes between 0.1 to 1 . In figure 2 the surface of the three dimensional plot shows maximums. The greatest maximum occurs at $f\left(k_{\max }^{\prime}, t_{o} / t_{\text {mcmax }}\right)=0.51$.

These maximums are local maximums.

In figure 3 the characteristic equation (44) based an proposal thread mill model ${ }^{7}$ is plotted. This plot shows no local maximums but there is a single maximum occurs at $t_{0} / t_{m c}=0, \mathrm{k}^{\prime}=10$ where $f\left(k^{\prime}, t_{0} / t_{m c}\right)=2.25$. Similar information could be obtained for equation (42) from level curves plotted by Dplot software presented at this work fig.4. This two dimensional plot is the image of three dimensional plot on the surface of the plan $t_{0} / t_{m c}=0.1, \mathrm{k}^{\prime}=1$.

Similar information could be obtained for equation (44) from level curves plotted by Dplot software presented at this work fig.5. This two dimensional plot is the image of three dimensional plot on the surface of the plane $t_{0} / t_{m c}=0.1, \mathrm{k}^{\prime}=1$.

Foley's characteristic equation $t_{o} / t_{m c}=0.1$ (46) has also been plotted three dimensionally figure 6 and two dimensionaly level curves figure 7. The information obtained from either these two plots is as following. Maximum $f\left(k^{\prime}, t_{o} / t_{m c}\right)=0.225$ occurs at $t_{o} / t_{m c}=0.1, \mathrm{k}^{\prime}=1$ and minimum $f\left(k^{\prime}, t_{o} / t_{m c}\right)=0$ occurs at $t_{o} / t_{m c}=1, \mathrm{k}^{\prime}=1$. 


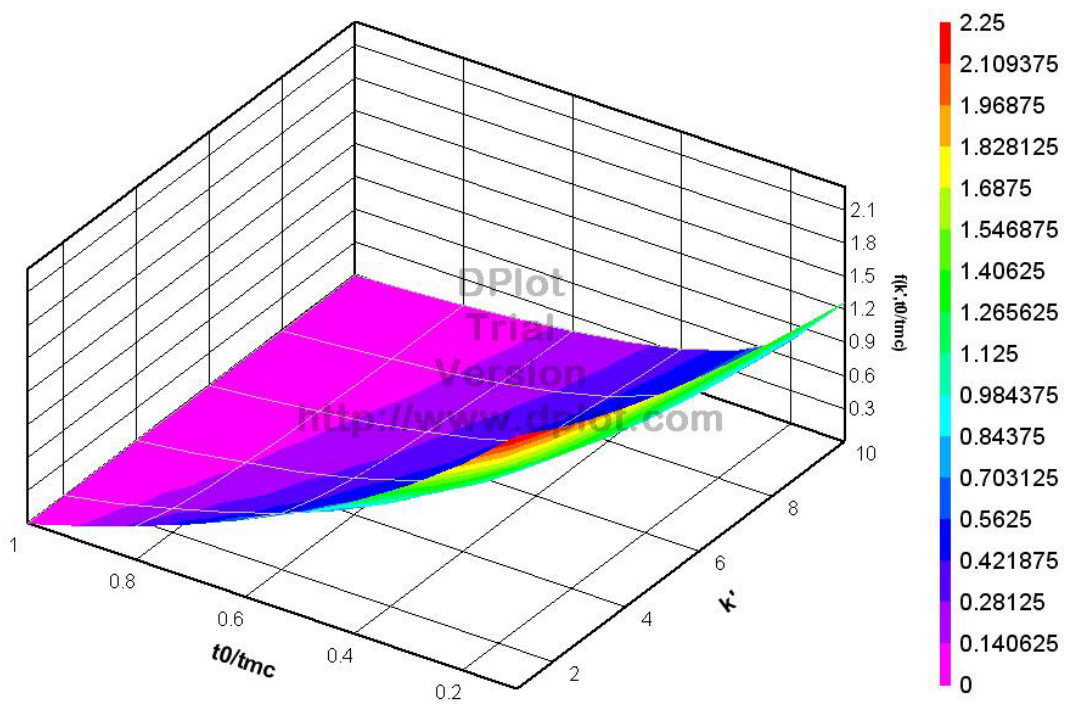

Figure 3. Three dimensional plot of Ghowsi's characteristic equation for resolution of MECC.

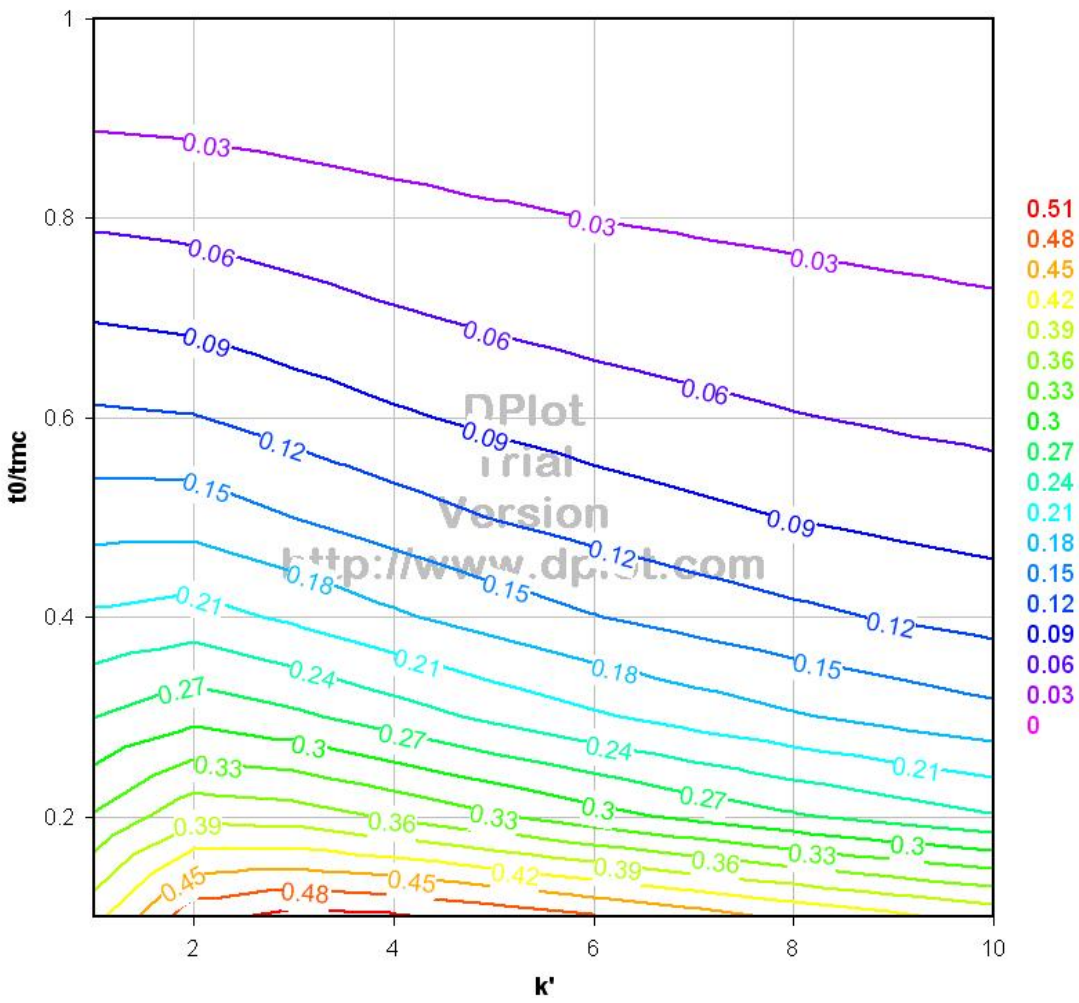

Figure 4. Two dimensional plot, level curves, of Terabe's characteristic equation for resolution of MECC. 


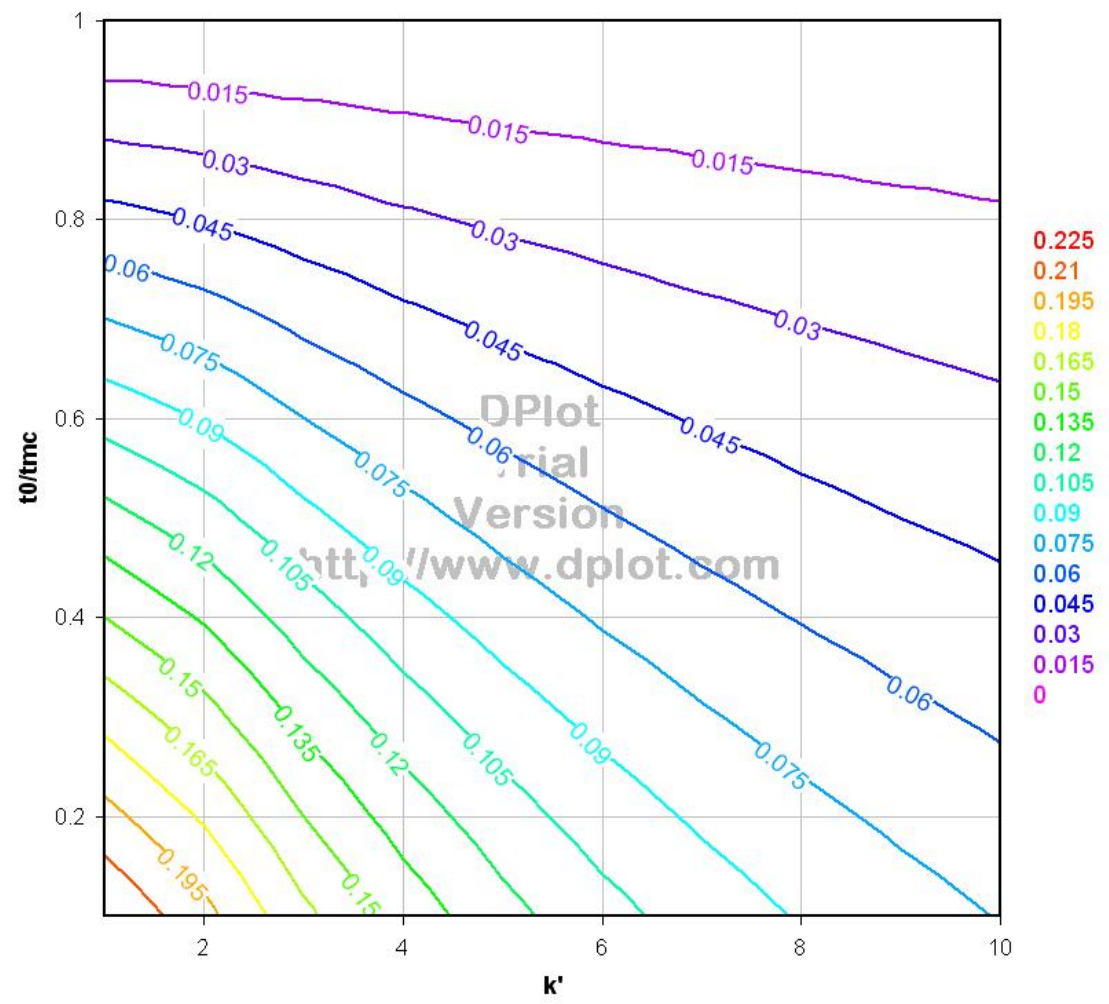

Figure 5. Two dimensional plot, level curves, of Ghowsi's characteristic equation for resolution of MECC.

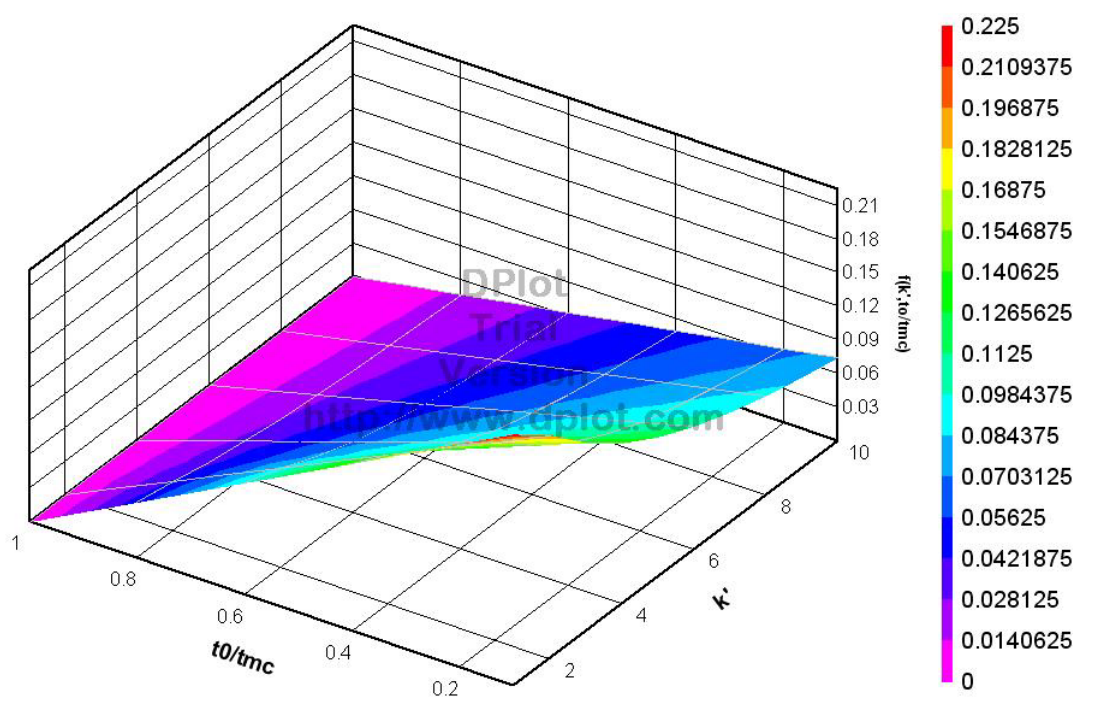

Figure 6. Three dimensional plot, Foley's characteristic equation for resolution per unit time $R_{s} / t_{R}$. 


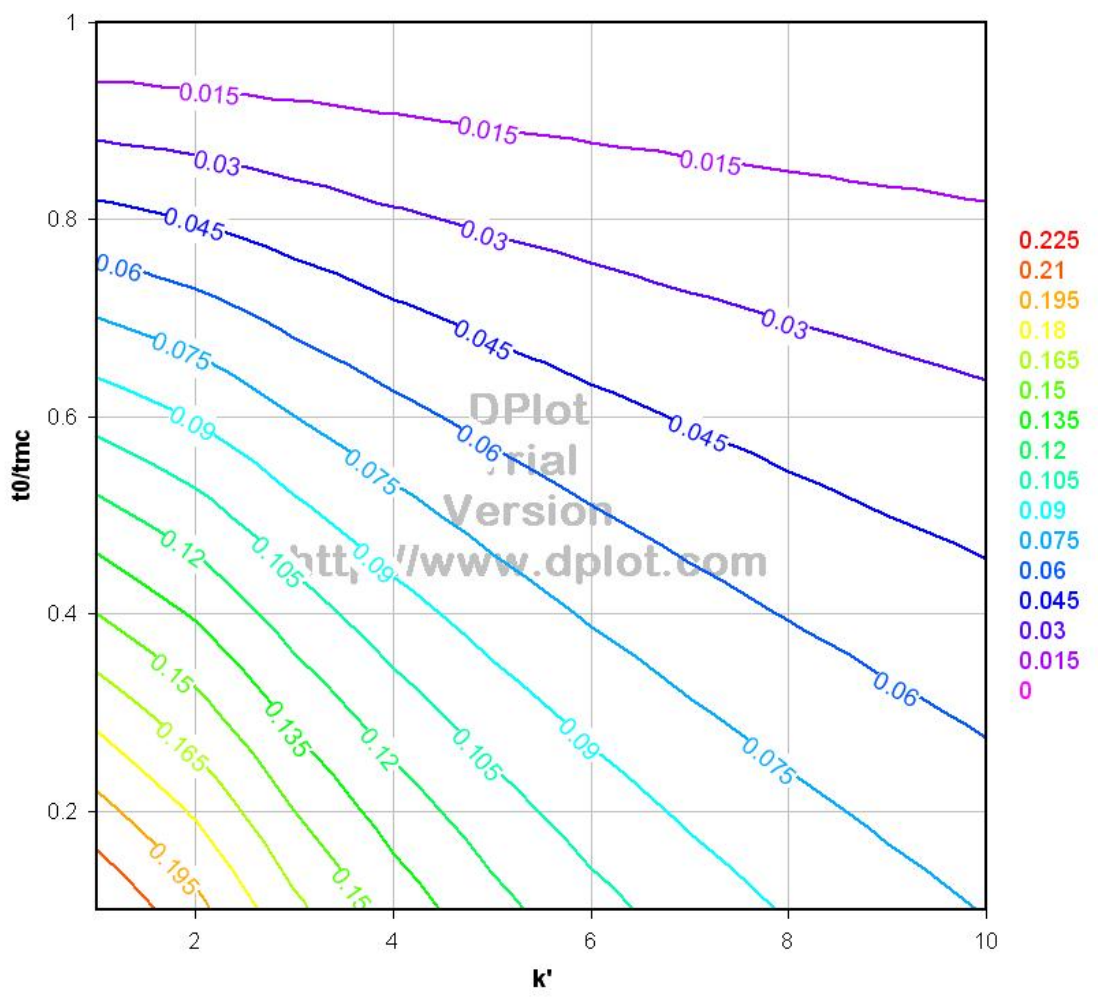

Figure 7. Two dimensional plot, level curves, of Foley's characteristic equation for resolution per unit time $R_{S} / t_{R}$.

Ghowsi's characteristic equation (47) has also been plotted three dimensionally figure 8 and two dimensional level curves figure 9. The information obtained from either these two plots is the following: Maximum $f\left(k^{\prime}, t_{o} / t_{m c}\right)=0.16$ occurs at $t_{o} / t_{m c}=0.1, \mathrm{k}^{\prime}=2$ and minimum $f\left(k^{\prime}, t_{o} / t_{m c}\right)=0$ occurs at $t_{o} / t_{m c}=1, \mathrm{k}^{\prime}=1$. 


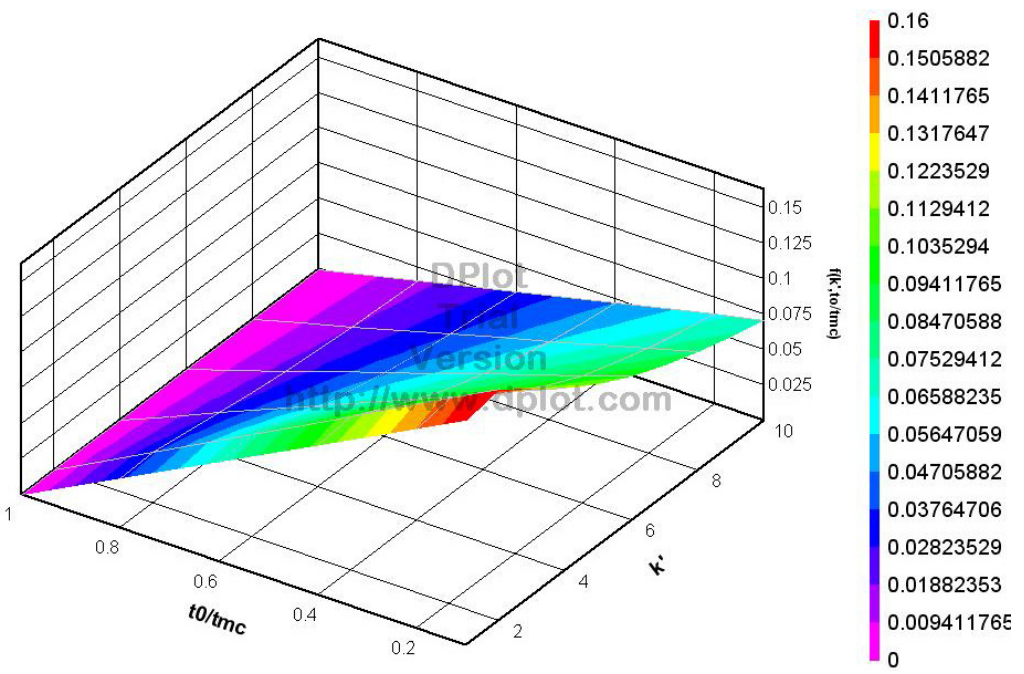

Figure 8. Tree dimensional plot of Ghowsi's characteristic equation for resolution per unit time $R_{s} / t_{R}$.

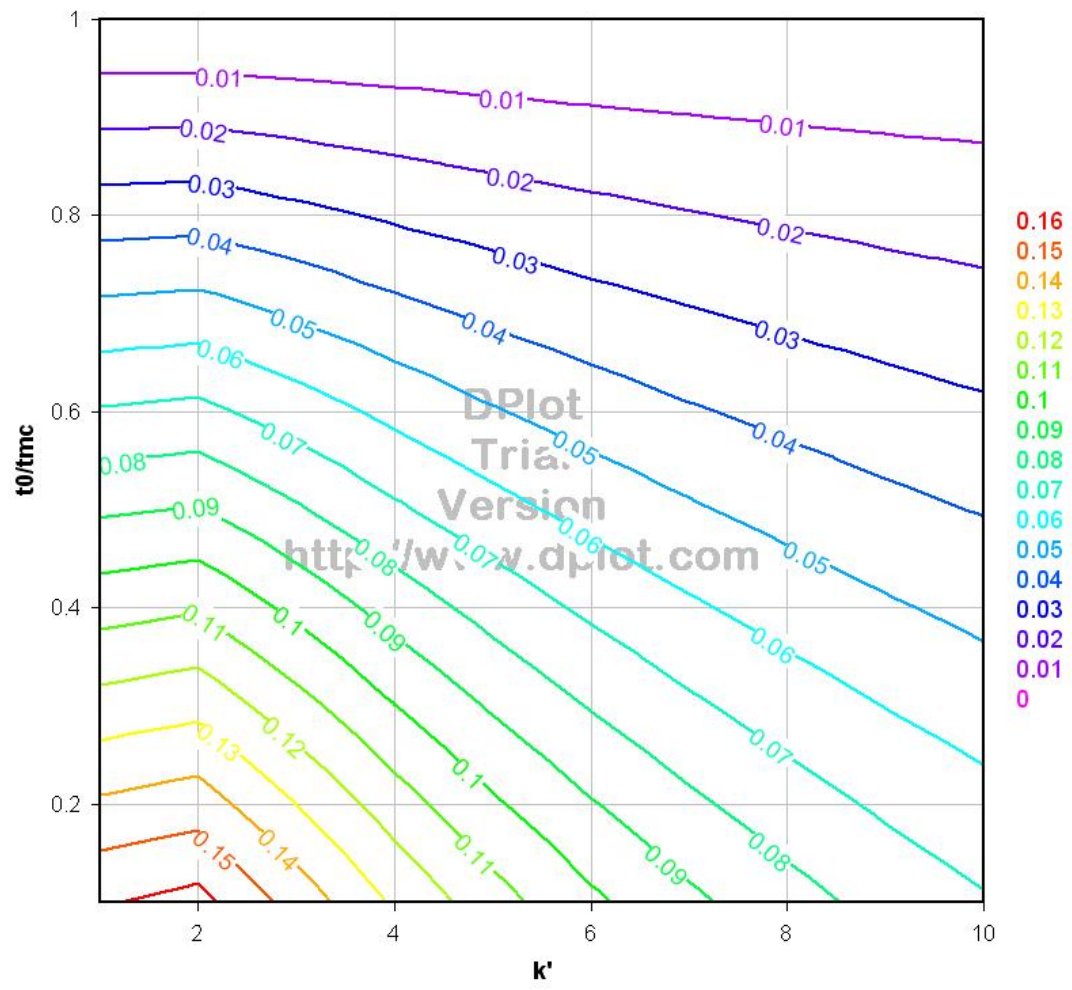

Figure 9. Two dimensional plot level curves of Ghowsi's characteristic equation for resolution per unit time $R_{s} / t_{R}$. 


\section{Conclusion}

The fundamental eqn.12 for the number of theoretical plates has been used to obtain resolution equation by Jorgenson eqn.14. The number of theoretical plates equation in previous work by Jorgenson is wrong, automatically makes the resolution equation wrong.

With the consideration that length $\mathrm{X}$ in eqn. 17 is the distance where force is applied in capillary electrophoresis, number of theoretical plates which depends on eqn.17 is discussed.

Another equation is the result obtained in eqn. 28 which indicates the new result based on effective length. In comparison with eqn.12 Jorgenson equation different eqn.26 has been used to obtain eqn.34 which is the new resolution equation. Similar procedure could be applied to obtain the number of theoretical plates for effective length for MECC and the new resolution equation could be obtained too. This work for MECC was shown in reference 7 .

In present work eight graph are presented as characteristic equations of MECC. Four of them are three dimensional and four of them are two dimensionals. It is very interesting observation that the Terabe's et al characteristic equation shows local maximums in three dimensional plots but the three dimensional plot of characteristic equation obtained by us based on thead mill model shows no local maximums on the surface of three dimensional plot.

\section{Author details}

Kiumars Ghowsi

Department of Chemistry Majlesi Branch, Islamic Azad University, Isfahan, I.R. Iran

Hosein Ghowsi

Department of Mathematics, Payame Noor University, Tehran, I.R. Iran

\section{References}

[1] J.C. Gidding, Sep.Sci.,4,181 (1969).

[2] J. Jorgenson and K.D. Lukacs, Anal. Chem., 53, 1298 (1981).

[3] C. D. Dunn, M. G. Hankins and K.Ghowsi , Sep.Sci. Tecnol. , 29,2419 (1994).

[4] W. R. Jones and J. Jandik, J. Chromatogr., 546, 445 (1991).

[5] K. Ghowsi, J. P. Foley and R. G. Gale, Anal. Chem.,62, 2714 (1990).

[6] D. A. Skoog, Principles of Instrumental Analysis, Saunder college Publishing Philadelphia, edn.5 (1992).

[7] K. Ghowsi and H. Ghowsi, Asian J. Chem., 23,3084 (2011).

[8] S. Terabe and T. Ando, Anal. Chem., 57,834 (1985). 
[9] J. P. Foley, Anal. Chem. 62,1302 (1990).

[10] K. Ghowsi and H. Ghowsi submitted to Asian. J. Chem. Accepted. 